\title{
Analysis of Defect-Related Inhomogeneous Electroluminescence in InGaN/GaN QW LEDs
}

\author{
C. X. Ren ${ }^{1}$, B. Rouet-Leduc ${ }^{1}$, J.T. Griffiths ${ }^{1}$, E. Bohacek ${ }^{1}$, M. J. Wallace ${ }^{2}$, P. R. Edwards ${ }^{2}$, M.

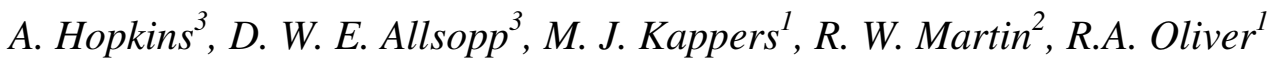

1. Department of Materials Science and Metallurgy, Charles Babbage Road, Cambridge, CB3 0FS, United

Kingdom

2. Department of Physics, SUPA, University of Strathclyde, Glasgow G4 0NG, United Kingdom

3. Department of Electronic and Electrical Engineering, University of Bath, Bath BA2 7AY, United Kingdom

\section{Abstract}

The inhomogeneous electroluminescence (EL) of $\mathrm{InGaN} / \mathrm{GaN}$ quantum well light emitting diode structures was investigated in this study. Electroluminescence hyperspectral images showed that inhomogeneities in the form of bright spots exhibited spectrally blue-shifted and broadened emission. Scanning electron microscopy combined with cathodoluminescence (SEM-CL) was used to identify hexagonal pits at the centre of approximately $20 \%$ of these features. Scanning transmission electron microscopy imaging with energy dispersive X-ray spectroscopy (STEM-EDX) indicated there may be $p$-doped AlGaN within the active region caused by the presence of the pit. Weak beam dark-field TEM (WBDF-TEM) revealed the presence of bundles of dislocations associated with the pit, suggesting the surface features which cause the inhomogeneous EL may occur at coalescence boundaries, supported by trends in the number of features observed across the wafer.

\section{Introduction}

$\mathrm{In}_{x} \mathrm{Ga}_{1-x} \mathrm{~N}$ quantum well (QW) structures are the foundation for many present-day optoelectronic devices such as light-emitting diodes (LEDs). The gradual shift towards highefficiency semiconductor lighting solutions is evidence of the substantial impact III-nitrides have had internationally since the demonstration of the first high brightness LED by Nakamura et al.[1]. Despite their widespread adoption in LED applications, III-nitride alloys still suffer from a plethora of material issues arising from heteroepitaxial growth on foreign substrates [2]. Chief amongst these is perhaps the presence of threading dislocations (TDs) which are the source of undesirable effects in LEDs such as large reverse bias leakage current [3] and non-radiative recombination [4], [5]. TDs have also been shown to open up into inverted pyramidal defects known as 'V-defects' [6] though it has been suggested that these defects might not be deleterious in nature [7], [8], [9].

In this study, we report the investigation of the cause of inhomogeneous electroluminescence (EL) which is occasionally observed in $c$-plane InGaN/GaN LEDs [10], [11], [12]. Specifically, we will investigate here LEDs which exhibit luminescent features of higher brightness than the surrounding material on application of a bias. Using multiple microscopy techniques to study these features has allowed us to correlate local emission properties in the LED to micro and nano-scale structural features and thus elucidate the cause of the inhomogeneous EL. 


\section{Materials and Methods}

The LED structures were grown by low-pressure metalorganic vapour-phase epitaxy (MOVPE) in a Thomas Swan $6 \times 2$-inch closed-coupled showerhead reactor. The structure studied consists of a low dislocation density (TD density ca. $5 \times 10^{8} \mathrm{~cm}^{-2}$ ) GaN template on sapphire with a $2 \mu \mathrm{m}$ layer of undoped GaN followed by a $3 \mu \mathrm{m}$ layer of silicon-doped GaN, a 5 period InGaN/GaN multiple quantum well $(\mathrm{MQW})$ region (well width $=3.9 \mathrm{~nm}$ ) with unintentionally doped GaN barriers $(7.6 \mathrm{~nm})$, a magnesium doped AlGaN electron blocking layer $(20 \mathrm{~nm})$ and a magnesium-doped GaN cap layer $(117 \mathrm{~nm})$. The thicknesses of the QW layers were determined from X-ray diffraction (XRD) measurements following the procedure of Vickers et al [13]. The QWs were grown using the twotemperature - "2T" - method described by Oliver et al. [14], in which reference full details of the growth of the LEDs may be found. The LEDs were $1 \times 1 \mathrm{~mm}^{2}$ side-contacted devices with interdigitated electrodes: processing and fabrication details have been described by Wallace et al [12]. The LEDs described in these two papers have a different QW thickness and do not display the same EL inhomogeneity reported here.

Images of the LED surfaces under application of a forward bias were taken in a Signatone S-1160 probe station. A total of 64 devices were imaged at different locations on the processed wafer.

EL hyperspectral images were collected in order to characterise the local optoelectronic properties at the inhomogeneities using a modified Cameca SX100 electron probe micro-analyser with a custom built cathodoluminescence set-up. EL measurements were performed with the electron beam blanked at a forward bias of $3 \mathrm{~V}$ and detected via an $8 \mu \mathrm{m}$ diameter pinhole in the collection path that enables a spatial resolution of approximately $3 \mu \mathrm{m}$.

Scanning electron microscopy with cathodoluminescence (SEM-CL) was performed on an FEI XL30 field emission gun SEM operating at $10 \mathrm{kV}$ at room temperature in panchromatic mode. Sample preparation for transmission electron microscopy (TEM) was performed using a dual beam focussed ion beam (FIB)/SEM FEI Company ${ }^{\mathrm{TM}}$ Helios Nanolab ${ }^{\mathrm{TM}}$ equipped with an Omniprobe Autoprobe ${ }^{\mathrm{TM}}$ 200 micro-manipulator using a standard in-situ lift-out procedure. The prepared samples were studied by scanning-TEM (STEM) using an FEI Company TM Tecnai Osiris with an extreme-FEG (X-FEG) at $200 \mathrm{kV}$, with energy dispersive X-ray (EDX) spectra recorded by four silicon drift detectors forming a solid angle greater than 0.9 sr to determine the chemical identities

Simulations of the LED under application of a forward bias were performed using the Crosslight APSYS simulation package which self-consistently computes the carrier transport equations with a quantum-mechanical model for photon emission from the QWs, with material parameters taken from Piprek [15]3. Results

A typical probe station EL image of an LED under a forward bias of $3 \mathrm{~V}$ is shown in Figure 1a). Bright features can be seen randomly distributed across the device. The number of bright features per LED was determined through the analysis of images taken from the EL of 64 different LEDs.

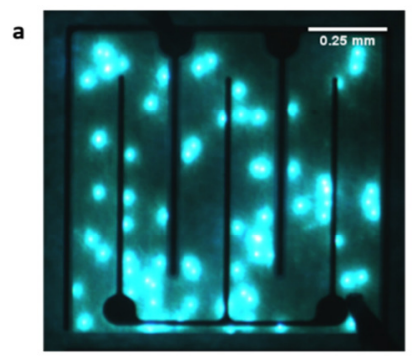

b

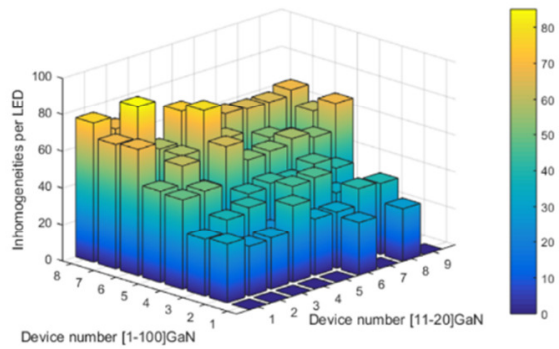

Figure 1: a) LED EL image taken at an injection current of $10 \mathrm{~mA}$ showing inhomogeneous features in the EL, b) Number of features per device for 64 devices. A trend is clear in the [11-20] GaN direction but not the $[1-100]_{\text {GaN }}$ direction. 
The number of features observed per device varied along the $[1-100]_{\mathrm{GaN}}$ direction, but not along the $[11-20]_{\mathrm{GaN}}$ direction, as shown in Figure $\left.1 \mathbf{b}\right)$. The $[1-100]_{\mathrm{GaN}}$ direction corresponds to the radial direction on the susceptor during device growth.

EL hyperspectral imaging was performed on several areas containing bright features to characterise their emission properties. Fig. 2 shows a representative data set. The features observed are of higher brightness, spectrally blue-shifted and broadened relative to the featureless background. The diameter of the observed features is typically between $5-10 \mu \mathrm{m}$.

a

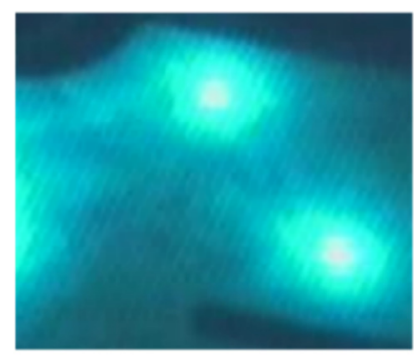

c

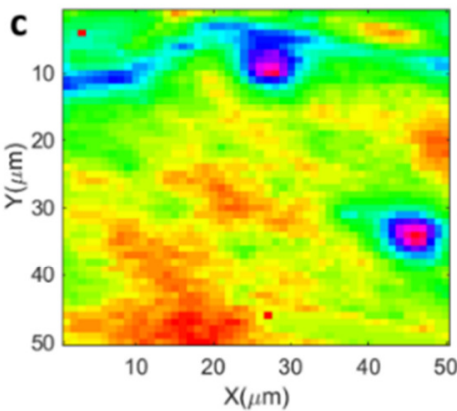

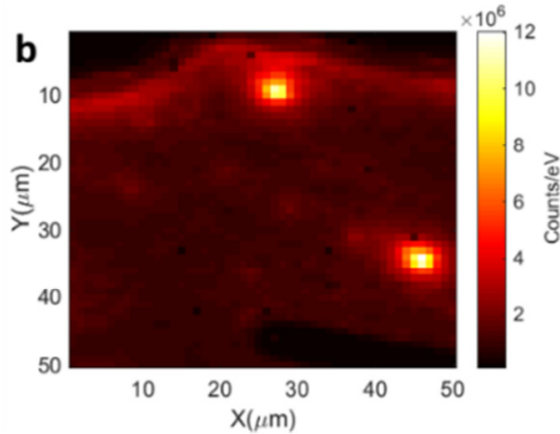

$$
\text { d }
$$

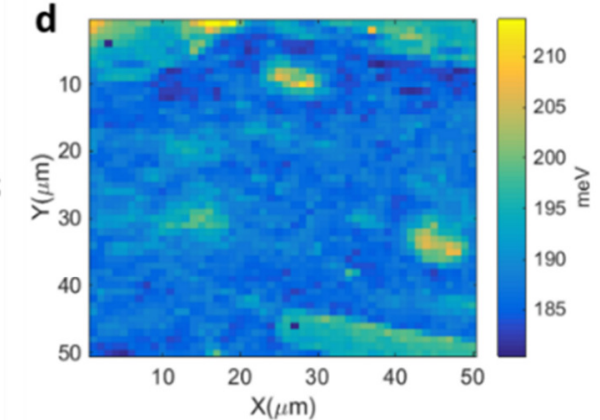

Figure 2:a) CCD image of features analysed by EL hyperspectral imaging, b) peak intensity, c) peak energy and d) emission FWHM of the features.

The features were also apparent when imaged in SEM-CL: concentric ring-like features of alternating high and low CL intensity were observed in the panchromatic CL image and in the concurrently recorded SEM image hexagonal pits were observed at the centre of approximately $20 \%$ of 765 features surveyed using this method. An example is shown in Fig. 3 where the location of the pit can be seen to be surrounded by a region of highly emitting material approximately $1 \mu \mathrm{m}$ across, followed by a ring of low CL intensity.
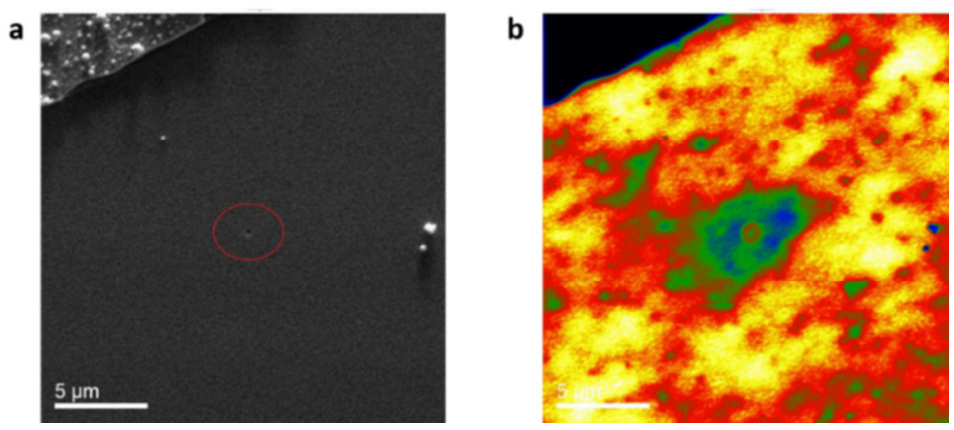

Figure 3: a) Secondary electron image of hexagonal pit (red circle) at the centre of the feature shown in the panchromatic $C L$ image taken in $b$ ). Colours in the panchromatic image are summed intensities with red being the highest and blue the lowest. The scale bars for both figures are identical. 
The identification of a hexagonal pit at the centre of our emission features allowed for the preparation of a TEM specimen by FIB lift-out of the centre of the feature with high accuracy. The hexagonal defect was visible during the milling process, providing a useful marker of the region of interest in the TEM lamella.

STEM-EDX was performed on an area of the lamella containing a portion of the defect in order to ascertain any compositional changes the defect may have induced in the LED structure. Fig. 4 b), c), d) show the compositional analysis of the EDX mapping in the area delineated by the red dashed line Fig .4a). In the indium (In) and gallium (Ga) maps (Figs 4 b) and d)) we see that the InGaN QWs are significantly disrupted by the hexagonal defect. Additionally, we observe the Al signal overlapping with the QW In signal, and the penetration of In below the QW stack along the slope of the pit.

a

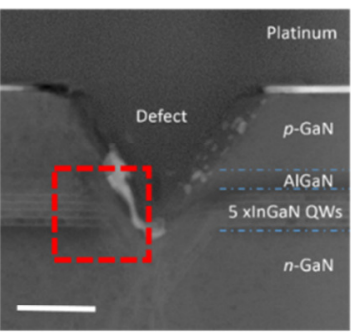

c

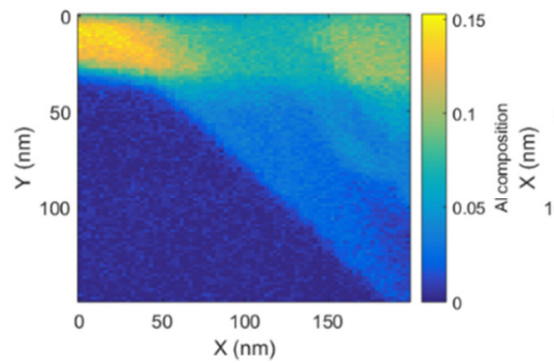

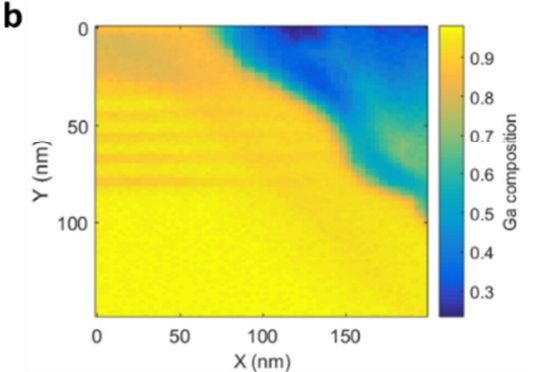

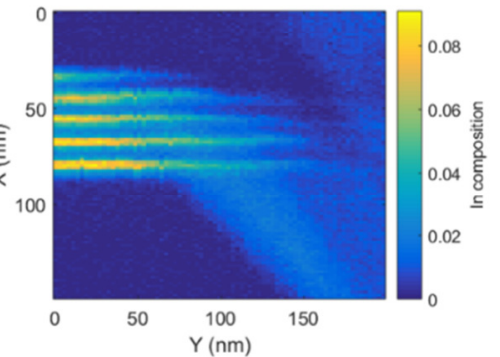

Figure 4: a) HAADF-STEM image of defect disrupting the active region of the LED structure, (150 nm scale bar). CliffLorimer analysis of the ternary composition: b) Ga composition c) Al composition and d) In composition.

In order to investigate what defects are associated with the hexagonal defect, weak beam dark field TEM was performed on the sample showing the presence of several looping dislocations, shown in Fig. 5.
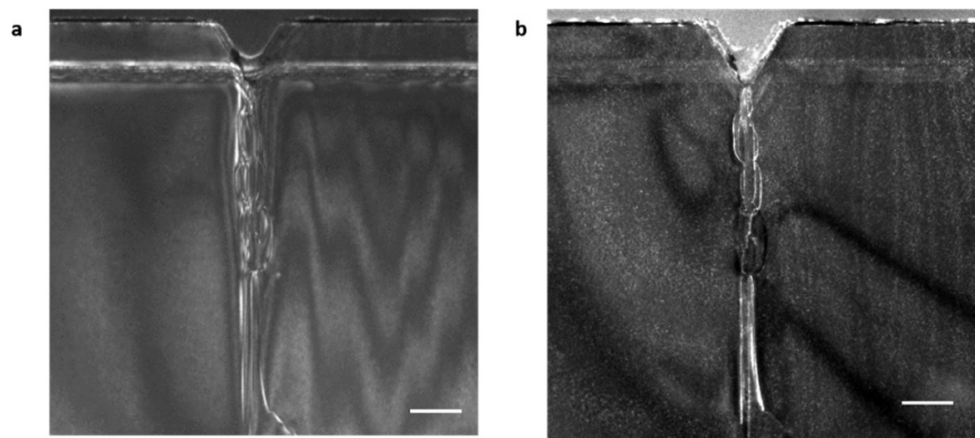

Figure 5;WBDF-TEM of multiple TDs associated with the hexagonal defect under (a) $g=<11 \overline{2} 0>$ (b) $g=<0001>.150$ nm scale bar. 


\section{Discussion}

\subsection{Origin of EL Inhomogeneity}

Fig. 4(c) shows the distortion of the active region layers due to the presence of a V-defect. We investigated the mechanisms by which the presence of the AlGaN laterally adjacent to the InGaN QWs associated with this hexagonal defect could influence the EL properties of the LED through the use of numerical simulations as described in [16]. The LED structure was simulated with similar dimensions to our devices, with the presence of the hexagonal defect simulated, as shown in Fig.6.

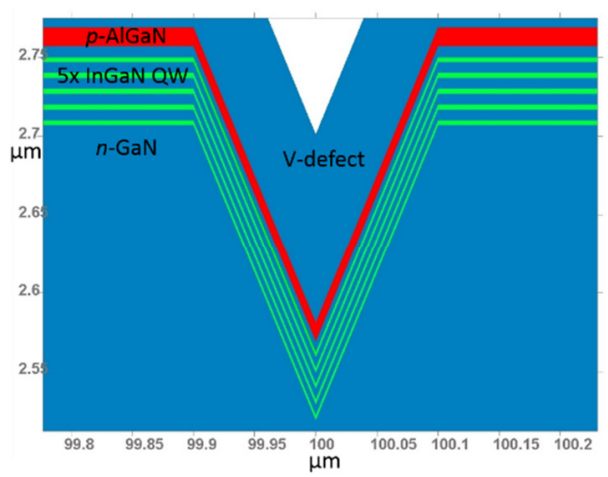

Figure 6: Simulated LED structure, with an incorporated hexagonal defect disrupting the active region.

The results of the simulation are shown below in Fig. 7. We find that the presence of the hexagonal defect does indeed result in an area with a high radiative recombination rate adjacent to the defect. The mechanism behind this effect can be ascertained by examining the electron and hole concentrations across the active region in area neighbouring the inclusion (red lines in Fig 7) and further away (black lines in Fig 7). The electron concentration (Fig 7 c) close to the inclusion remains largely unchanged relative to the surroundings, whereas the hole concentration in the deepest QW (Fig 7 (d)) is greatly enhanced, resulting in the enhanced radiative recombination rate as shown in Fig. 7. b) 
a
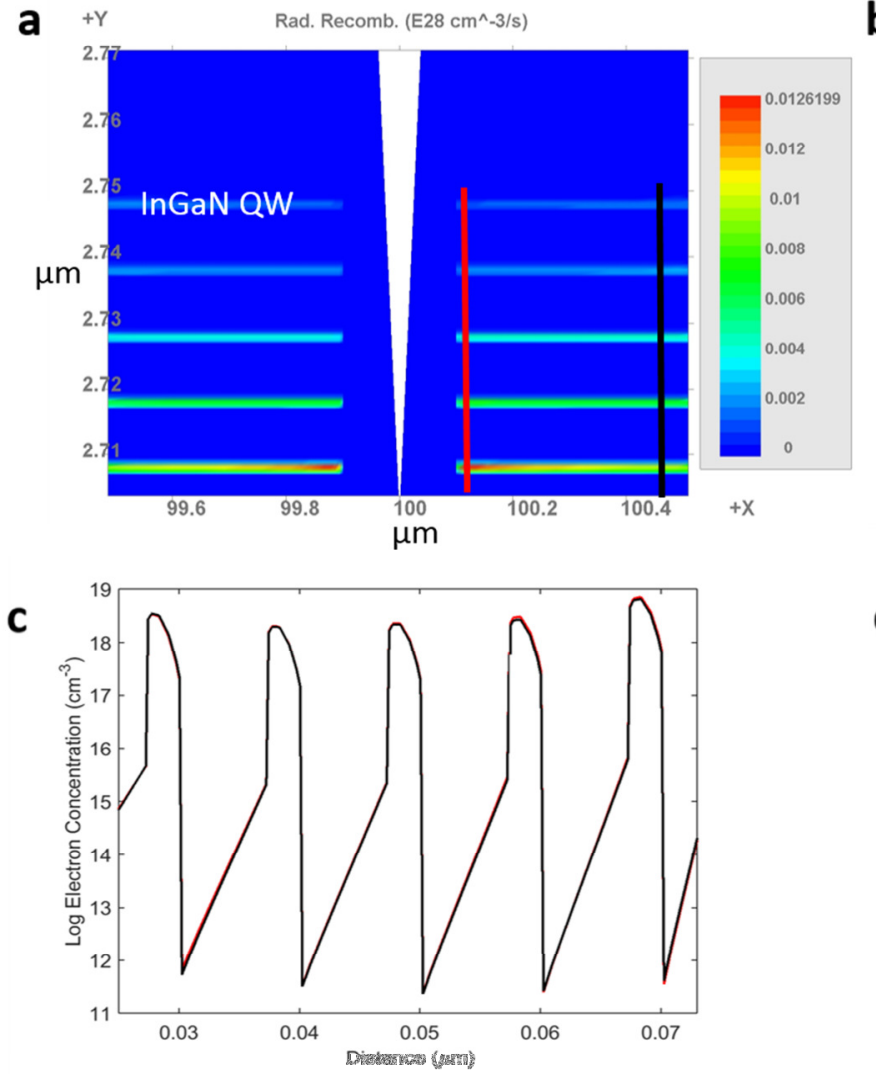

b

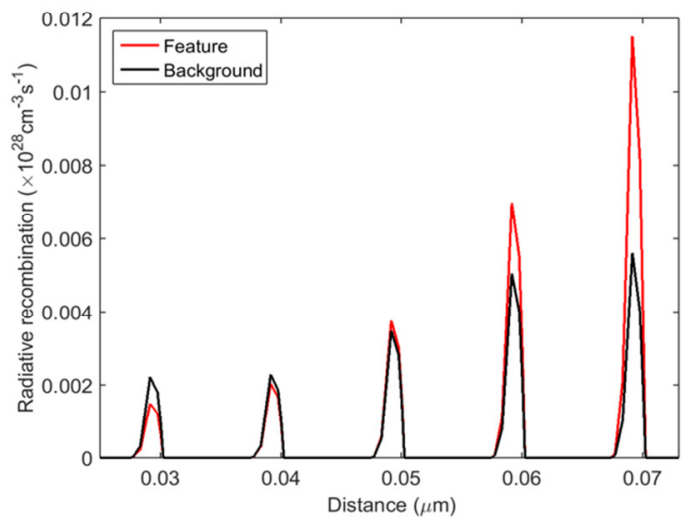

d

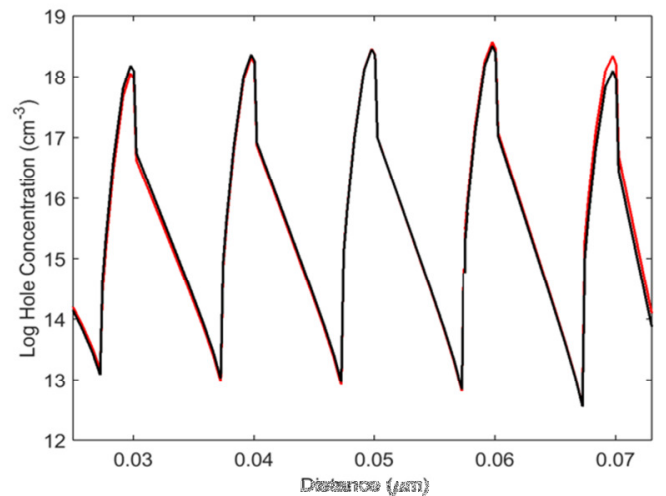

Figure 7: APSYS simulation results a) Radiative recombination events $b$ ) radiative recombination profiles $c$ ) electron concentration d) hole concentration. Red and black traces correspond to the profiles shown in a).

As such, the numerical simulation suggests the presence of this type of inhomogeneity in the EL can be ascribed to the increased hole injection in the local area surrounding the defect due to the influence of an effective lateral heterojunction being formed in the MQW as a result of the presence of AlGaN in the hexagonal defect. This interpretation is supported by the hyperspectral EL data shown in Fig.2: the spectral blue shift observed in Fig. 2 (c) and the emission line-width broadening observed in Fig. 2 (d) both of which may be due to valence band filling near the V-pit by the excess hole concentration. Whilst variations in strain and composition around the pit could also influence the blue-shift, we note that the blue shift in EL is low at small injection currents and rises with increasing current, which supports the suggestion drawn from the model that this is a key effect. It is however important to note that the contribution of the bundle of TDs to local conductivity [8],[17] has not been incorporated in this model. We also note that the lateral dimension and relative brightness of the feature depend on the $p$-doping of the AlGaN sidewalls of the defect, however the feature is present across a range of dopant concentrations examined in our model.

\subsection{Origin of the Hexagonal Defect}

Typical V-defects are associated with a single TD [18], and are known to be present at all TD sites in QW samples of the type presented here, initiating at the first QW, but are filled on p-capping. The hexagonal defect we discuss is not a typical V-defect since it is associated with a bundle of threading dislocations (Fig 5). This bundle of TDs might be attributed to vertical side facets in GaN grains [19] during the original growth of the GaN template on sapphire. Dislocations tend to bend towards island side facets and then on coalescence may annihilate one another or propagate upwards in a bundle [19]. A similar effect is also seen in epitaxial laterally overgrown (ELOG) GaN samples at 
the coalescence boundary of the ELOG wings [20]. Thus, one explanation of our observation of large hexagonal pits would be imperfect coalescence at grain boundaries resulting in a cluster of dislocations nucleating this form of defect. Although such hexagonal pits are only observed in approximately $20 \%$ of the bright features examined in the SEM-CL, it is possible that other similar pits fill up during the growth of the final $p$-GaN cap [8]. This is consistent with the data shown in Fig. 2. since temperature gradients often arise in the MOVPE reactor along the direction in which the number of high brightness features per LED device varies, and such a temperature gradient could lead to variations in the rate of island coalescence [21] and hence to the number of hexagonal pits occurring. This result shows that, even if the average dislocation density is relatively low, nonuniformity in their distribution can still severely degrade the electroluminescence of InGaN/GaN LEDs.

\section{Conclusions}

In conclusion, we have studied both the spectral and structural properties of bright features in the electroluminescence of $c$-plane InGaN/GaN MQW LEDs. Hyperspectral EL hyperspectral images reveal that the features are not only brighter than the background, but spectrally blue-shifted and broadened. STEM-EDX shows the presence of Al penetrating through the active region due to the hexagonal defect. Simulations performed suggest that the mechanism behind the bright features is the enhanced injection of holes to the active region due to the $\mathrm{AlGaN}$ inclusion, but it is important to note that other mechanisms may also make significant contributions to the predicted effects. WBDF-TEM imaging was used to show the presence of multiple dislocations associated with the apex of the Vdefect, suggesting the bright features appear at coalescence boundaries. Overall, our analysis suggests that careful control of $\mathrm{GaN}$ template growth at the coalescence stage is key to avoiding such lateral inhomogeneity in the EL from these devices.

\section{Acknowledgements}

The European Research Council has provided financial support under the European Community's Seventh Framework Programme/ ERC grant agreement no. 279361 (MACONS).

\section{References}

[1] S. Nakamura, T. Mukai, and M. Senoh, "High-Power GaN P-N Junction Blue-Light-Emitting Diodes," Jpn. J. Appl. Phys., vol. 30, no. 12A, pp. 1998-2001, 1991.

[2] F. C.-P. Massabuau, S.-L. Sahonta, L. Trinh-Xuan, S. Rhode, T. J. Puchtler, M. J. Kappers, C. J. Humphreys, and R. A. Oliver, "Morphological, structural, and emission characterization of trench defects in InGaN/GaN quantum well structures," Appl. Phys. Lett., vol. 101, no. 21, p. 212107, 2012.

[3] S. E. Bennett, "Dislocations and their reduction in GaN," Mater. Sci. Technol., vol. 26, no. 9, pp. 1017-1028, 2010.

[4] M. Albrecht, J. L. Weyher, B. Lucznik, I. Grzegory, and S. Porowski, "Nonradiative recombination at threading dislocations in n-type GaN: Studied by cathodoluminescence and defect selective etching," Appl. Phys. Lett., vol. 92, no. 23, pp. 0-3, 2008. 
[5] D. Cherns, S. J. Henley, and F. A. Ponce, "Edge and screw dislocations as nonradiative centers in InGaN/GaN quantum well luminescence," Appl. Phys. Lett., vol. 78, no. 18, pp. 2691-2693, 2001.

[6] S. E. Bennett, D. Holec, M. J. Kappers, C. J. Humphreys, and R. a Oliver, "Imaging dislocations in gallium nitride across broad areas using atomic force microscopy.," Rev. Sci. Instrum., vol. 81, no. 6, p. 063701, Jun. 2010.

[7] A. Hangleiter, F. Hitzel, C. Netzel, D. Fuhrmann, U. Rossow, G. Ade, and P. Hinze, "Suppression of nonradiative recombination by $\mathrm{V}$-shaped pits in GaInN/GaN quantum wells produces a large increase in the light emission efficiency," Phys. Rev. Lett., vol. 95, no. 12, pp. $1-4,2005$.

[8] S. H. Han, D. Y. Lee, H. W. Shim, J. Wook Lee, D. J. Kim, S. Yoon, Y. Sun Kim, and S. T. Kim, "Improvement of efficiency and electrical properties using intentionally formed Vshaped pits in InGaN/GaN multiple quantum well light-emitting diodes," Appl. Phys. Lett., vol. 102, no. 25, 2013.

[9] Y. Li, F. Yun, X. Su, S. Liu, W. Ding, and X. Hou, "Deep hole injection assisted by large Vshape pits in InGaN/GaN multiple-quantum-wells blue light-emitting diodes," J. Appl. Phys., vol. 116, no. 12, p. 123101, 2014.

[10] Y. Lin, Y. Zhang, Z. Liu, L. Su, J. Zhang, T. Wei, and Z. Chen, "Spatially resolved study of quantum efficiency droop in InGaN light-emitting diodes," Appl. Phys. Lett., vol. 101, no. 25, p. 252103, 2012.

[11] M. J. Wallace, P. R. Edwards, M. J. Kappers, M. A. Hopkins, F. Oehler, S. Sivaraya, D. W. E. Allsopp, R. A. Oliver, C. J. Humphreys, and R. W. Martin, "Bias dependence and correlation of the cathodoluminescence and electron beam induced current from an InGaN/GaN light emitting diode," J. Appl. Phys., vol. 116, no. 3, 2014.

[12] M. J. Wallace, P. R. Edwards, M. J. Kappers, M. A. Hopkins, F. Oehler, S. Sivaraya, R. A. Oliver, C. J. Humphreys, D. W. E. Allsopp, and R. W. Martin, "Effect of the barrier growth mode on the luminescence and conductivity micron scale uniformity of InGaN light emitting diodes," J. Appl. Phys., vol. 117, no. 11, p. 115705, 2015.

[13] M. E. Vickers, M. J. Kappers, T. M. Smeeton, E. J. Thrush, J. S. Barnard, and C. J. Humphreys, "Determination of the indium content and layer thicknesses in InGaN/GaN quantum wells by x-ray scattering," J. Appl. Phys., vol. 94, no. 3, pp. 1565-1574, 2003.

[14] R. A. Oliver, F. C.-P. Massabuau, M. J. Kappers, W. A. Phillips, E. J. Thrush, C. C. Tartan, W. E. Blenkhorn, T. J. Badcock, P. Dawson, M. A. Hopkins, D. W. E. Allsopp, and C. J. Humphreys, "The impact of gross well width fluctuations on the efficiency of GaN-based light emitting diodes," Appl. Phys. Lett., vol. 103, no. 14, p. 141114, 2013.

[15] J. Piprek, Nitride Semiconductor Devices: Principles and Simulation, January 20. Wiley-VCH, 2007.

[16] J. Piprek, "Origin of InGaN/GaN light-emitting diode efficiency improvements using tunneljunction-cascaded active regions," Appl. Phys. Lett., vol. 104, no. 5, pp. 2-6, 2014. 
[17] Q. Shan, D. S. Meyaard, Q. Dai, J. Cho, E. Fred Schubert, J. Kon Son, and C. Sone, "Transport-mechanism analysis of the reverse leakage current in GaInN light-emitting diodes," Appl. Phys. Lett., vol. 99, no. 25, p. 253506, 2011.

[18] H. K. Cho, J. Y. Lee, G. M. Yang, and C. S. Kim, "Formation mechanism of V defects in the $\mathrm{InGaN} / \mathrm{GaN}$ multiple quantum wells grown on $\mathrm{GaN}$ layers with low threading dislocation density," Appl. Phys. Lett., vol. 79, no. 2, pp. 215-217, 2001.

[19] R. Datta, M. J. Kappers, M. E. Vickers, J. S. Barnard, and C. J. Humphreys, "Growth and characterisation of GaN with reduced dislocation density," Superlattices Microstruct., vol. 36, no. 4-6, pp. 393-401, Oct. 2004.

[20] A. Sakai, H. Sunakawa, A. Kimura, and A. Usui, "Self-organized propagation of dislocations in GaN films during epitaxial lateral overgrowth," Appl. Phys. Lett., vol. 76, no. 4, p. 442, 2000.

[21] S. Das Bakshi, J. Sumner, M. J. Kappers, and R. A. Oliver, "The influence of coalescence time on unintentional doping in GaN/sapphire," J. Cryst. Growth, vol. 311, no. 2, pp. 232-237, Jan. 2009. 